\title{
Shock Wave Mitigation by Air Plasma Deflector
}

\author{
Spencer P. Kuo \\ Department of Electrical \& Computer Engineering, New York University-Tandon School of Engineering, Brooklyn, NY, USA \\ Email: spk259@nyu.edu
}

How to cite this paper: Kuo, S.P. (2018) Shock Wave Mitigation by Air Plasma Deflector. Advances in Aerospace Science and Technology, 3, 71-88.

https://doi.org/10.4236/aast.2018.34006

Received: October 20, 2018

Accepted: December 17, 2018

Published: December 20, 2018

Copyright (c) 2018 by author and Scientific Research Publishing Inc. This work is licensed under the Creative Commons Attribution International License (CC BY 4.0).

http://creativecommons.org/licenses/by/4.0/

\begin{abstract}
When the spacecraft flies much faster than the sound speed $(\sim 1200 \mathrm{~km} / \mathrm{h})$, the airflow disturbances deflected forward from the spacecraft cannot get away from the spacecraft and form a shock wave in front of it. Shock waves have been a detriment for the development of supersonic aircrafts, which have to overcome high wave drag and surface heating from additional friction. Shock wave also produces sonic booms. The noise issue raises environmental concerns, which have precluded routine supersonic flight over land. Therefore, mitigation of shock wave is essential to advance the development of supersonic aircrafts. A plasma mitigation technique is studied. A theory is presented to show that shock wave structure can be modified via flow deflection. Symmetrical deflection evades the need of exchanging the transverse momentum between the flow and the deflector. The analysis shows that the plasma generated in front of the model can effectively deflect the incoming flow. A non-thermal air plasma, generated by on-board $60 \mathrm{~Hz}$ periodic electric arc discharge in front of a wind tunnel model, was applied as a plasma deflector for shock wave mitigation technique. The experiment was conducted in a Mach 2.5 wind tunnel. The results show that the air plasma was generated symmetrically in front of the wind tunnel model. With increasing discharge intensity, the plasma deflector transforms the shock from a welldefined attached shock into a highly curved shock structure with increasing standoff distance from the model; this curved shock has increased shock angle and also appears in increasingly diffused form. In the decay of the discharge intensity, the shock front is first transformed back to a well-defined curve shock, which moves downstream to become a perturbed oblique shock; the baseline shock front then reappears as the discharge is reduced to low level again. The experimental observations confirm the theory. The steady of the incoming flow during the discharge cycle is manifested by the repeat of the baseline shock front.
\end{abstract}

\section{Keywords}

Shock Wave Mitigation, Electric Discharge, Air Plasma Deflector, 
Shadowgraph, Drag Reduction, Wind Tunnel, Charge Transfer

\section{Introduction}

Shock wave appears in the form of a steep pressure gradient. It introduces a discontinuity in the flow properties at the shock front location. The background pressure behind the shock front increases considerably, leading to significant enhancement of the flow drag and friction on the spacecraft. Thus, the design for high-speed aircraft tends to choose slender shapes to reduce the drag and cooling requirements. This is an engineering tradeoff between volumetric and fuel consumption efficiencies and this tradeoff significantly increases the operating cost of commercial supersonic aircraft. Shock wave also produces sonic booms. The noise issue raises environmental concerns, which have precluded routine supersonic flight over land.

Theoretical and experimental efforts have been devoted to the understanding of shock waves in supersonic flows [1]. Various approaches to reduce shock wave drag have been explored. A physical spike [2] is currently used in a supersonic spacecraft to move shock wave upstream from the spacecraft. It improves the body aspect ratio of a blunt-body and significantly reduces the wave drag. Further mitigation of shock wave impact calls for the development of new technologies, which attenuates or even eliminates shock wave formation around a supersonic vehicle. The payoff includes the reduction of fuel consumption and having smaller propulsion system requirements for the same cruise speed.

Thermal energy deposition in front of a flying body to perturb the incoming flow and shock wave formation has been studied [3] [4] [5] [6] [7]. The heating of the gas in front of a flying body essentially reduces the Mach number of the incoming flow in the heated region. This weakens shock wave and increases the shock angle (i.e., moving the shock front upstream). However, it requires a large heating power [5] to significantly elevate the gas temperature in order for this approach to effectively reduce the wave drag and shock noise in the supersonic flows. In fact, the energy gain from drag reduction is not enough to make up the injected heating energy. Although this is not a practical approach for drag reduction purposes, it can be an easy approach for sonic boom attenuation [3].

Non-thermal modification effects of plasmas on the shock wave structures have been evidenced in a number of shock-tube experiments. The study of plasma mitigation of shock waves is further inspired by the observation of a wind tunnel experiment conducted by Gordeev et al. [8]. By exploding a high Z conducting wire, inside the chamber of a cone-cylinder model, off electrical short circuit, a high-pressure metal vapor (high Z) plasma was produced and injected into the upstream supersonic flow through a nozzle. A significant drag reduction, which was too large to be accounted for by the thermal effect alone, was measured. In the subsequent wind tunnel experiments [9]-[17], electric discharges were applied to generate plasmas in the supersonic flows to interact with 
the shock waves. The results showed that the shock front increased dispersion in its structure and/or the standoff distance from the model when the plasma was generated either off-board or on-board ahead of a model. Computational and experimental studies [18] indicate that an added magnetic field can strengthen the arc plasma to further weaken the shock wave. However, microwave plasma projected on-board was shown still too weak to introduce any visible effect on the shock wave in a hypersonic flow [19].

A long-lasting plasma effect on the shock structure (i.e., it takes much longer than the discharge period, after the discharge ceases, to recover to the baseline state) was also observed in the experiments by Baryshnikov et al. [9] and by Bivolaru and Kuo [13] [14], who investigated the relaxation time of the modified shock wave structure in decaying discharge plasma. To further advance the development of the plasma mitigation technique, a better understanding the plasma mitigation mechanism is necessary and is explored in the present work.

Theory shows that the shock wave angle and the shock structure depend on the cone angle of the wind tunnel model, and on the Mach number and the deflection angle of the incoming flow [1]. In the present study, the cone angle of the wind tunnel model and the Mach number of the incoming flow are fixed, an on-board plasma deflector is introduced to study shock structure modification by the flow deflection [20]. The polarity of the applied voltage enables electron plasma to be accelerated in the upstream direction by the applied electric field, it forms a plasma deflector in the upstream region to deflect incoming flow through elastic collisions. Ion plasma also affects the incoming flow, but via a different process. Ions moving through their own gas are subject to charge transfer to the neutral gas, which is a predominant inelastic collision process in the low ion energy regime [21]. An ion becomes a neutral particle after the charge transfer, but retains its velocity, which is usually low. Thus, these particles do not contribute to the shock wave formation. The ions converted from neutrals through charge-transfer are collected by the cathode and do not contribute to the shock wave generation either. The shock of the deflected flow is expected to have a larger shock angle (than that of the baseline one) and a modified structure, representing a weaker shock.

In Section 2, the Taylor-Maccoll's theory for a normally incident supersonic flow over a cone is generalized to the case of obliquely incident flow. A plasma deflector generated by an electric discharge is modeled and the flow deflection by this deflector is formulated in Section 3. Numerical illustration of the plasma deflection effect is presented in Section 4. Wind tunnel experimental results to demonstrate shock mitigation by a plasma deflector, generated by $60 \mathrm{~Hz}$ periodic arc discharge, are presented in Section 5. In Section 6, a summary of the work is given. Conclusion remarks are drawn in Section 7.

\section{Supersonic Flow over a Cone}

Starting with a simple situation that the incoming supersonic flow from the left 
propagates along the axis of a cone. In the steady state, a conic shock front signified by a step pressure jump is formed to separate the flow into regions 1 and 2 of distinct entropies as sketched in Figure 1 with $\theta^{\prime}=0$. In the figure, the cone is placed horizontally (along the z-axis); thus, the flow velocity $V_{1}$ in region 1 is along the $z$ (cone's) axis, i.e., $\boldsymbol{V}_{1}=V_{1} \hat{\boldsymbol{a}}_{z}=V_{1}\left(\hat{\boldsymbol{a}}_{R} \cos \beta-\hat{\boldsymbol{a}}_{\theta} \sin \beta\right)$, where $\hat{\boldsymbol{a}}_{R}$ and $\hat{\boldsymbol{a}}_{\theta}$ are unit vectors in the radial and poloidal directions of the spherical coordinate system, the origin is at the tip of the cone. The flow has a Mach number $M_{1}$. The conic shock wave angle $\beta$ is to be determined. In region 2 immediately behind the shock front, the flow has a deflection angle $\delta$ with Mach number $M_{2}$ and velocity $\boldsymbol{V}_{2}=V_{1}\left[\hat{\boldsymbol{a}}_{R} \cos (\beta-\delta)-\hat{\boldsymbol{a}}_{\theta} \sin (\beta-\delta)\right]$. Across an oblique shock wave, the continuity of the flow, i.e., $\rho_{1} V_{1} \sin \beta=\rho_{2} V_{2} \sin (\beta-\delta)$ where $\rho$ is the mass density of the flow, together with the preservation of the tangential component (i.e. $\hat{\boldsymbol{a}}_{R}$ component) of the flow velocity, i.e., $V_{1} \cos \beta=V_{2} \cos (\beta-\delta)$, relate the shock wave angle $\beta$ and the deflection angle $\delta$ through a $\delta-\beta-M$ relation [1] [22]:

$$
\tan \delta=2 \cot \beta\left\{\left(M_{1}^{2} \sin ^{2} \beta-1\right) /\left[M_{1}^{2}(\gamma+\cos 2 \beta)+2\right]\right\}
$$

where $\gamma=1.4$ is usually adopted.

Taylor-Maccoll's theory is applied to analyze a deflected supersonic flow over a cone. The incoming flow from the left is now to propagate at a constant angle $\theta^{\prime}$ with respect to the axis of the cone as sketched in Figure 1. We first extend the $\delta-\beta-M$ relation (1) to $\theta^{\prime} \neq 0$ situation. The changes across a conic shock wave (similar to across an oblique shock) are governed by the normal component of the free-stream velocity, the relevant parameters in the equations are $M_{n 1}=M_{1} \sin \left(\beta-\theta^{\prime}\right)$ and $M_{n 2}=M_{2} \sin (\beta-\delta)$. Letting $\beta^{\prime}=\left(\beta-\theta^{\prime}\right)$ and

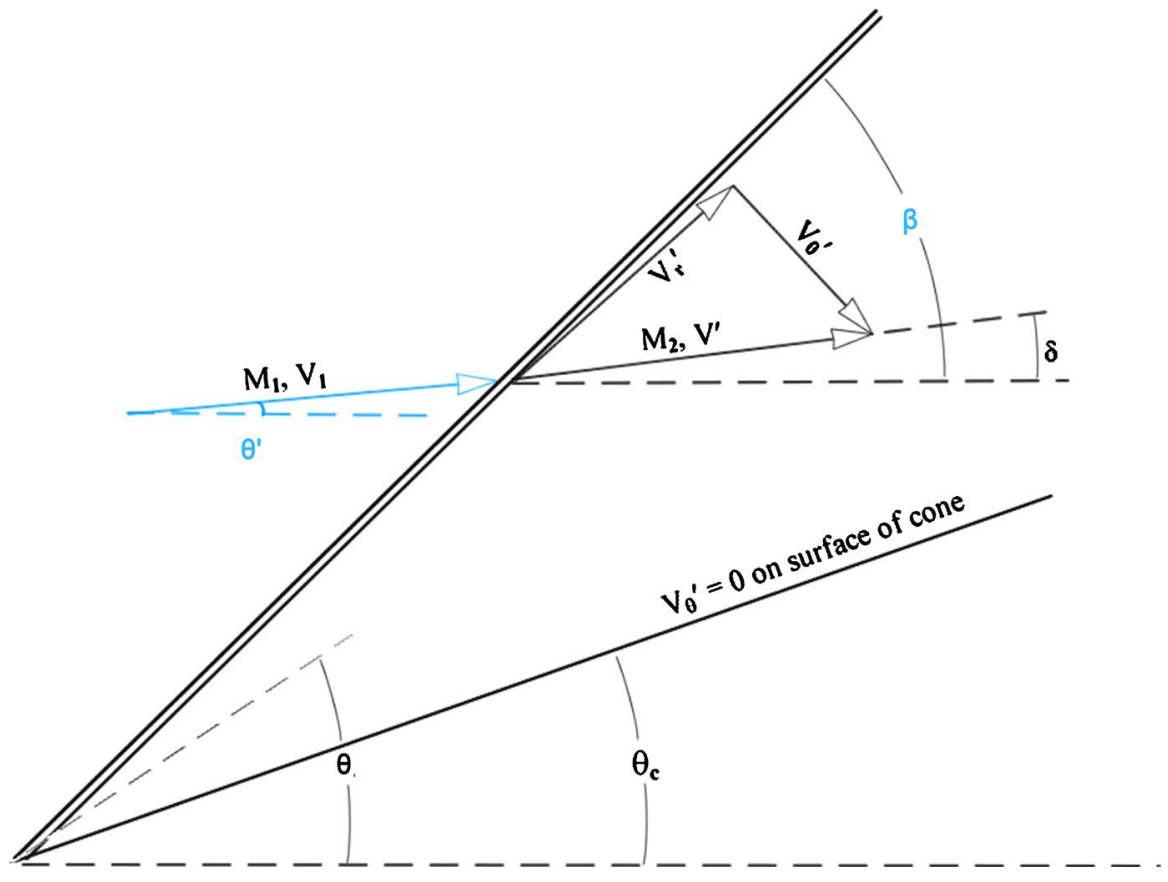

Figure 1. Geometry for the numerical solution of deflected flow over a cone. 
$\delta^{\prime}=\left(\delta-\theta^{\prime}\right)$, these two relations become $M_{n 1}=M_{1} \sin \beta^{\prime}$ and $M_{n 2}=M_{2} \sin \left(\beta^{\prime}-\delta^{\prime}\right)$, which are expressions similar to those in the $\theta^{\prime}=0$ case. In essence, this is to rotate the $z$ axis counterclockwise by an angle $\theta^{\prime}$. Therefore, in the case of $\theta^{\prime} \neq 0$, the $\delta-\beta-M$ relation is extended to the $\delta^{\prime}-\beta^{\prime}-M$ relation [17] [22] to be

$$
\tan \delta^{\prime}=2 \cot \beta^{\prime}\left\{\left(M_{1}^{2} \sin ^{2} \beta^{\prime}-1\right) /\left[M_{1}^{2}\left(\gamma+\cos 2 \beta^{\prime}\right)+2\right]\right\}
$$

The normalized Taylor-Maccoll equation for conical flows (Eq. 10.15 of Anderson [1]) is expressed as

$$
0.2\left[1-G^{2}-G^{\prime 2}\right]\left[2 G+G^{\prime} \cot \theta+G^{\prime \prime}\right]-G^{\prime 2}\left[G+G^{\prime \prime}\right]=0
$$

where $G=V_{R 2} / V_{2 \max }, G^{\prime}=\mathrm{d} G / \mathrm{d} \theta, G^{\prime \prime}=\mathrm{d}^{2} G / \mathrm{d} \theta^{2}$, and $\gamma=1.4$ is assumed.

The boundary conditions of (3), at the poloidal angle $\theta=\beta$, are given by

$$
\begin{aligned}
& G(\beta)=f\left(M_{2}\right) \cos (\beta-\delta)=f\left(M_{2}\right) \cos \left(\beta^{\prime}-\delta^{\prime}\right) \\
& G^{\prime}(\beta)=-f\left(M_{2}\right) \sin (\beta-\delta)=-f\left(M_{2}\right) \sin \left(\beta^{\prime}-\delta^{\prime}\right)
\end{aligned}
$$

where $f\left(M_{2}\right)=V_{2} / V_{2 \max }=\left[\left(5 / M_{2}^{2}\right)+1\right]^{-1 / 2}, M_{2}=M_{n 2} / \sin \left(\beta^{\prime}-\delta^{\prime}\right)$, $M_{n 2}=\left\{\left[\left(M_{1} \sin \beta^{\prime}\right)^{2}+5\right] /\left[7\left(M_{1} \sin \beta^{\prime}\right)^{2}-1\right]\right\}^{1 / 2}$, and $\delta^{\prime}$ is determined by (2). Thus $G(\theta ; \beta)$ and $G^{\prime}(\theta ; \beta)$ can be evaluated for a given $M_{1}$ and $\beta$. If $\beta$ represents the wave angle of the shock on a $\theta$ half-angle cone, an additional boundary condition that the normal component of the flow velocity on the cone surface is zero, i.e., $G^{\prime}(\theta ; \beta)=0$, has to be imposed.

Equation (3) can be solved for $G\left(\theta_{c} ; \beta_{c}\right)$, where $\theta_{c}$ and $\beta_{c}$ are the half-cone angle and the wave angle of the shock on the cone, via a direct or an inverse approach. The direct numerical approach is to continuously adjust the boundary conditions by varying $\beta$ until $G^{\prime}\left(\theta_{c} ; \beta_{c}\right)=0$ with a proper boundary angle $\beta=\beta_{c}$. From this, the wave angle $\beta_{c}$ of the shock on a $\theta_{c}$ half-angle cone is determined. However, it makes the calculation easy by employing an inverse approach; a given shock wave will be assumed and the particular cone that supports the given shock will be calculated.

That is setting $\beta=\beta_{c}^{\prime}$, a given shock wave angle $\beta_{c}^{\prime}$, in (4) to solve (3) for $G\left(\theta ; \beta_{c}^{\prime}\right)$ and $G^{\prime}\left(\theta ; \beta_{c}^{\prime}\right)=0$ until $G^{\prime}\left(\theta=\theta_{c}^{\prime} ; \beta_{c}^{\prime}\right)=0$. Thus a plot of $\beta_{c}^{\prime}$ verse $\theta_{c}^{\prime}$ can be obtained; from which $\left(\theta_{c}, \beta_{c}\right)$ is determined. The effect of a localized plasma deflector on the shock wave is then inferred from changes in the deflection angle $\theta^{\prime}$ and in the Mach number $M_{1}$ of the flow, where $\theta^{\prime}$ and $M_{1}$ vary with $r$, the radial coordinate with respect to the $\mathrm{z}$-axis.

At a fixed $M_{1}$, the deflection angle $\delta$ and the shock wave angle $\beta$, in the case of $\theta^{\prime}=0$, increase only with the half-cone angle $\theta_{c}$. This is illustrated in Figure 2(a) and Figure 2(b). As the cone angle exceeds a critical value, i.e., $\theta_{c}>\theta_{c m}$, the solution of (3) cannot meet the boundary condition that the normal component of the flow velocity on the cone surface is zero any more.

Thus, the shock will become detached and the oblique shock becomes a curved shock, as shown in Figure 2(c). On the other hand, in the case of $\theta^{\prime} \neq 0$, 
the deflection angle $\delta$ and the shock wave angle $\beta$ increase with the half-cone angle $\theta_{c}$ as well as with the deflection angle $\theta^{\prime}$ of the incoming flow. It suggests that the shock wave angle of a fixed cone can be increased by introducing a symmetric deflection on the incoming flow; and further increase of the deflection can even modify the attached oblique shock to a detached curve shock. The impact of the flow deflection on the shock wave modification is illustrated in Figure 3, showing in sequence the envisioned shock wave change with the increase of the flow deflection. Therefore, if a plasma generated in front of the cone tip can effectively deflect the incoming flow symmetrically, it can mitigate shock wave impact by increasing the shock wave angle and changing oblique shock into detached curve shock. A very significant deflection of the incoming flow may even impede the shock wave formation.

\section{Plasma Deflection}

To deflect the incoming flow effectively, it favors that plasma is generated in the region upstream of the baseline shock front and has a symmetrical spatial distribution with respect to the axis of a cone [23]. In the following discussion, a symmetrically distributed plasma generated in front of the base-line shock [22] [23] [24] [25] as the plasma deflector is assumed.

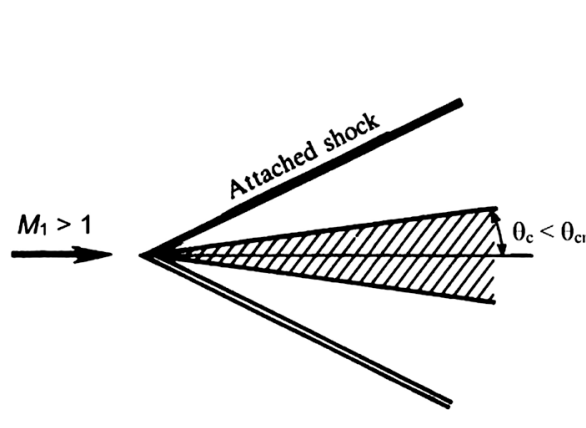

(a)

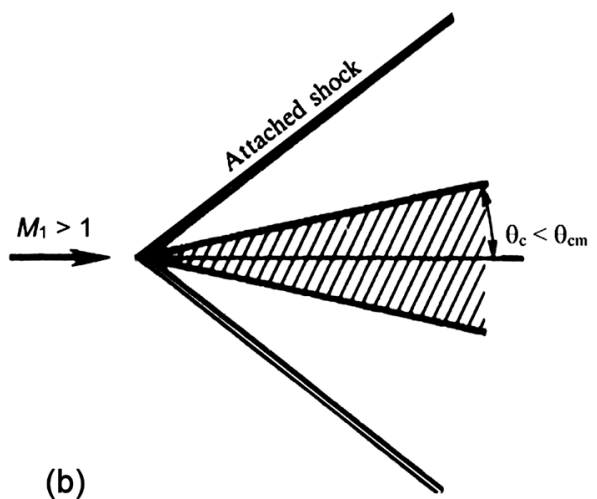

(b)

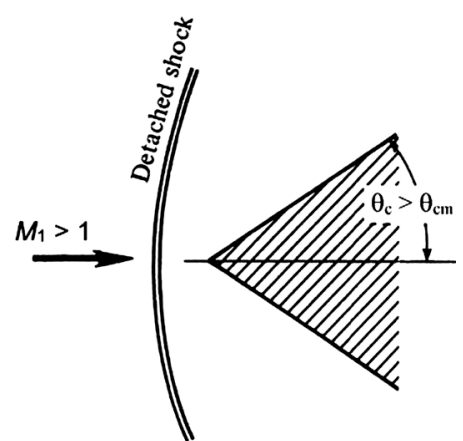

(c)

Figure 2. (a) Attached oblique shock wave of a cone with $\theta_{c}<\theta_{c m}$; (b) Increased shock angle with the increase of the cone angle; (c) Changed to a detached curve shock as $\theta_{c}>\theta_{c m}$.

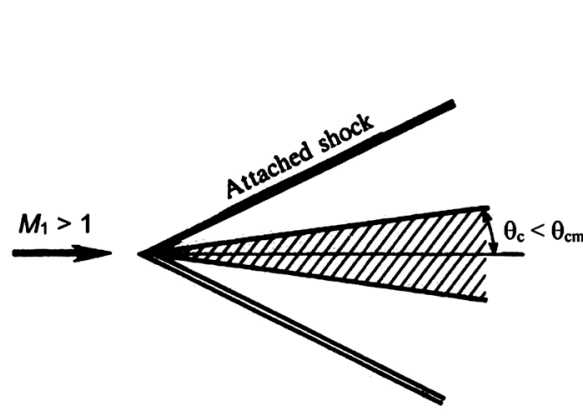

(a)
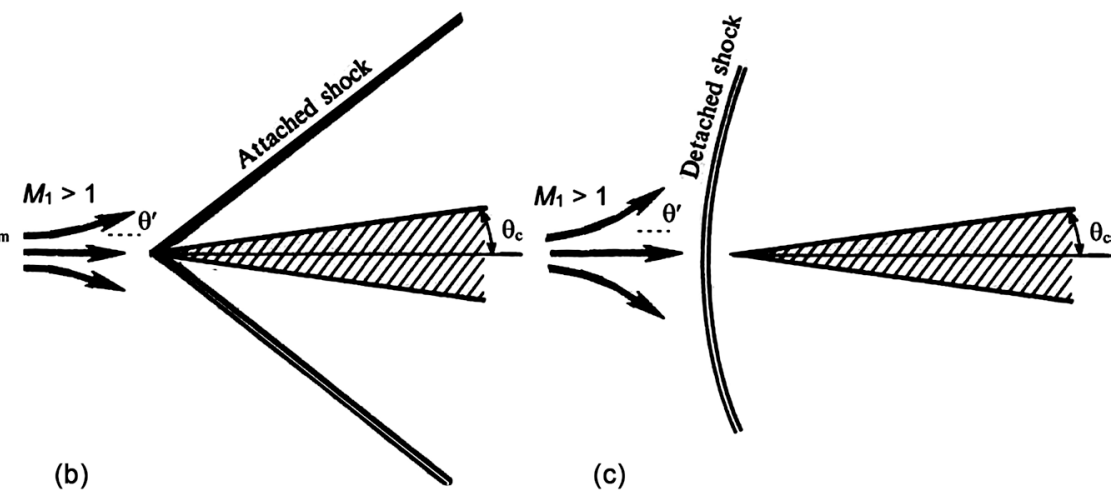

(c)

Figure 3. A shock wave (a) On a cone with $\theta_{c}<\theta_{c m}$ is modified by the degree of the incoming flow deflection, (b) Increase of the shock angle as the deflection angle $\theta^{\prime}<\theta_{m}^{\prime}$, and (c) Becoming a detached curve shock as $\theta^{\prime}>\theta_{m}^{\prime}$. 


\subsection{Plasma Deflector}

This plasma deflector is introduced at a location in front of a (cone-shaped) wind tunnel model by an on-board electrical discharge, which is triggered by a negative voltage applied between the grounded body of the cone and the tip of the cone located at $z=0$, which is insulated from the body. The sharpness of the tip helps to enhance the electric field intensity in the region in front of the tip. Using the planar projection of the model as a two-dimensional model, the equipotential lines between the two electrodes, with the central electrode biased negatively, are evaluated numerically by using a Poisson solver. The result is presented in Figure 4(a), in which the distribution of the electric field that is perpendicular to the equipotential line is also indicated. As shown, the applied electric field can extend to a relatively large region upstream of the baseline shock front, but the electric field intensity decreases with distance from the tip. This local field continuously accelerates electrons in the upstream region to increase their kinetic energy. Some of them will gain enough energy for ionization, and the remaining will deflect the incoming flow via momentum transfer collisions. The transverse momentum perturbation of the neutral flow is distributed symmetrically in opposite directions. A cartoon figure to illustrate the flow deflection is presented in Figure 4(b), in which the electric field pushing electrons upstream is modeled to be $\boldsymbol{E}(r, z) \cong-\left(A_{0} / R^{2}\right)(z \hat{\boldsymbol{z}}+r \hat{\boldsymbol{r}})$, where $(r, z)$ are the cylindrical coordinates, $A_{0}$ is proportional to the applied voltage and $R=\left(z^{2}+r^{2}\right)^{1 / 2}$ is the distance of the field point away from the tip. Because electrons collide the incoming flow much more frequently than ions and the local field, in the region upstream of the tip of the model, accelerates ions in the direction of the flow, electrons deflect the flow stronger than the ions. The deflection is most effective when plasma has a symmetric distribution around the tip, so that the net change

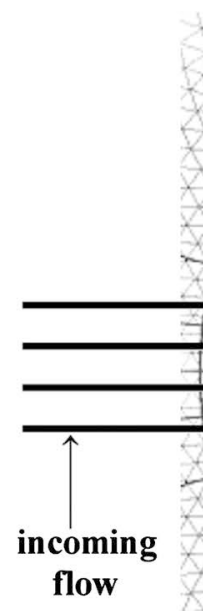

flow field lines (a) deflected by plasma
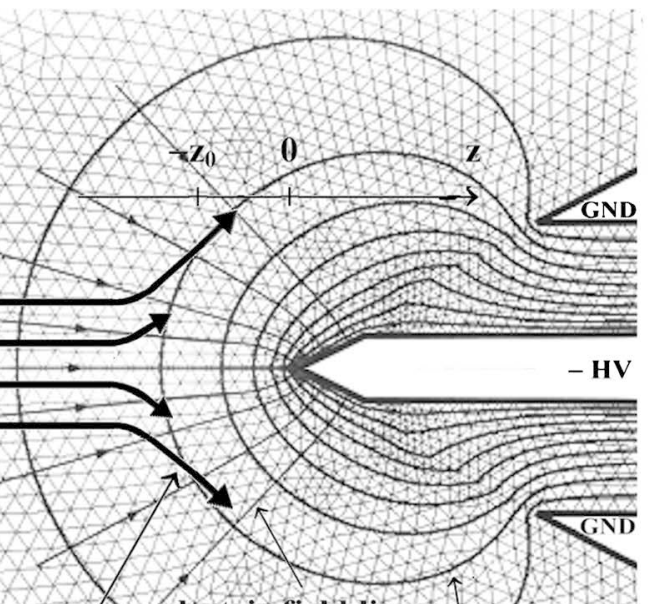

electric field lines

Figure 4. (a) Distributions of the equipotential line and electric field between two electrodes of a two-dimensional model and (b) A cartoon showing the envisioned electric field distribution supporting the discharge and the deflection of the incoming flow by the discharge-produced plasma. 
of the total momentum of the deflected flow in the transverse direction is zero; thus this transverse perturbation can be large even in the situation that the electron mass is much smaller than the masses of the neutral particles in the flow.

We now consider that a uniform airflow from left to right with a velocity $\boldsymbol{V}_{10}=V_{10} \hat{\boldsymbol{z}}$ encounters this plasma deflector at $z=-z_{0}$, as demonstrated in Figure 4(b). Electrons in the discharge interact with the airflow through elastic electron-neutral collisions. On the other hand, the ion-neutral interaction is more complicate. It involves both elastic and charge transfer inelastic collisions.

The electron density distribution of the plasma deflector is first determined through the spatial distribution of the ionization frequency $v_{i} \sim \varepsilon^{5.3} v_{a}$, where $\varepsilon=E / E_{c r}, E_{c r}$ is the air breakdown threshold field, and $v_{a}$ is the electron-neutral attachment rate. Thus $n_{e}(\xi)=n_{0} \exp \left[\left(v_{i}-v_{a}\right) t_{0}\right]=n_{e 0} \exp \left\{-\eta\left[1-\left(1+\xi^{2}\right)^{-2.65}\right]\right\}$, where $\xi=r / z_{0}, t_{0}$ is the transient period for the plasma density to build up, $n_{e 0}=n_{e}(0)=n_{0} \exp \left[\left(v_{i 0}-v_{a}\right) t_{0}\right], v_{i 0}=v_{i}(\xi=0), \quad \eta=v_{i 0} t_{0}$, and $\eta=0.85$ will be assumed. The two electric field components in the interaction region at $z=-z_{0}$ are represented approximately by $E_{z}=E_{0} /\left(1+\xi^{2}\right)$ and $E_{r}=-E_{0} \xi /\left(1+\xi^{2}\right)$, where $E_{0}=A_{0} / z_{0}$.

\subsection{Flow Deflection}

The momentum equations for the three fluids: electrons, positive ions and neutral molecules, in a weakly ionized plasma in the presence of an imposed electric field are:

$$
\begin{gathered}
m_{e} \mathrm{~d}\left(n_{e} \boldsymbol{v}_{e}\right) / \mathrm{d} t=-n_{e} m_{e} v_{e n}\left(\boldsymbol{v}_{e}-\boldsymbol{V}_{1}\right)+n_{e} m_{e} v_{e i}\left(\boldsymbol{v}_{i}-\boldsymbol{v}_{e}\right)-e n_{e} \boldsymbol{E} \\
m_{i} \mathrm{~d}\left(n_{i} \boldsymbol{v}_{i}\right) / \mathrm{d} t=-n_{i} m_{i} v_{i n}\left(\boldsymbol{v}_{i}-\boldsymbol{V}_{1}\right)-n_{i} m_{i} v_{c}\left(\boldsymbol{v}_{i}-\boldsymbol{V}_{10}\right)-n_{e} m_{e} v_{e i}\left(\boldsymbol{v}_{i}-\boldsymbol{v}_{e}\right)+e n_{i} \boldsymbol{E} \\
m_{n} \mathrm{~d}\left(n_{n} \boldsymbol{V}_{1}\right) / \mathrm{d} t=n_{e} m_{e} v_{e n}\left(\boldsymbol{v}_{e}-\boldsymbol{V}_{1}\right)+n_{i} m_{i} v_{i n}\left(\boldsymbol{v}_{i}-\boldsymbol{V}_{1}\right)+n_{i} m_{i} v_{c}\left(\boldsymbol{v}_{i}-\boldsymbol{V}_{10}\right)
\end{gathered}
$$

where $v_{c}\left(>v_{i n}\right)$ is the ion-neutral charge transfer collision frequency, which is dominated by charge transferring between the same type particles, e.g., the charge transfer cross-section between $\mathrm{N}_{2}^{+}$and $\mathrm{N}_{2}$ in the relevant energy regime is larger than $3 \times 10^{-19} \mathrm{~m}^{2}$. After charge transfer, neutral particle converts to an ion which moves at the neutral's original velocity $V_{10}$; and the ion converts to a neutral moving at ion's original velocity $\boldsymbol{V}_{\mathbb{D}}$. Since the ion's velocity is low, the converted neutrals form a subsonic flow, which will not contribute to the shock wave formation. On the other hand, the converted ions form a supersonic flow; but this ion flow will be collected by the cathode to close the discharge current loop, and will not contribute to the shock wave formation. Therefore, each of the ion and neutral fluids can be decomposed into two components, i.e., $n_{i} v_{i}=n_{i 1} v_{i}^{(1)}+n_{i 2} v_{i}^{(2)}$ and $n_{n} V_{1}=n_{n 1} V_{1}^{(1)}+n_{n 2} V_{1}^{(2)}$, where $n_{i 1}$ and $n_{i 2}$ are the ion densities generated by the electric discharge and by the charge exchange conversion, respectively; $n_{n 1}$ and $n_{n 2}$ are the neutral density of the incoming flow and the neutral density converted from the ions, respectively; thus $\mathrm{d} n_{i 2} / \mathrm{d} t=\mathrm{d} n_{n 2} / \mathrm{d} t=v_{c} n_{i 1}$; and 
$v_{i}^{(1)} \sim v_{i 0}, v_{i}^{(2)} \sim V_{10}, V_{1}^{(1)} \sim V_{10}$, and $V_{1}^{(2)} \sim v_{i 0}$. In other words, the one with superscript 1 is related to shock formation, and the other, with superscript 2, is not related to shock formation. $n_{n 2} \ll n_{n 1}$ because the interaction region (plasma layer) is very narrow. To simplify the analysis, the inertial terms on the left hand side and the electron-ion collision terms (proportional to $v_{e i}$ and $v_{i e}$ ) on the right hand side of (5) and (6) are neglected; the approximated (5) and (6) yield $n_{e} m_{e} v_{e n}\left(\boldsymbol{v}_{e}-\boldsymbol{V}_{1}^{(1)}\right) \cong-n_{e} e \boldsymbol{E}$ and $n_{i 1} m_{i} v_{i n}\left(\boldsymbol{v}_{i}^{(1)}-\boldsymbol{V}_{1}^{(1)}\right) \cong\left[v_{i n} /\left(v_{i n}+v_{c}\right)\right] n_{i 1}\left(e \boldsymbol{E}-m_{i} v_{c} \boldsymbol{V}_{1}^{(1)}\right)$, which reduce (7) to be

$\mathrm{d} \boldsymbol{V}_{1}^{(1)} / \mathrm{d} t=-\left[v_{c} /\left(v_{i n}+v_{c}\right)\right]\left(n_{e} / n_{n}\right) e \boldsymbol{E} / m_{n}-\left(n_{i 1} / n_{n}\right) v_{c}\left[\boldsymbol{V}_{10}+v_{i n} \boldsymbol{V}_{1}^{(1)} /\left(v_{i n}+v_{c}\right)\right]$

where $n_{i 1} \cong n_{e}$ is assumed.

It is noted that in (8) the pressure gradient term is neglected by assuming that the density and temperature of the airflow do not change considerably during the transit period of the airflow passing through the plasma deflector. We now integrate (8) over a transit period $t_{n}=z_{0} / V_{10}$, the time for the airflow to pass through the plasma deflector of length $z_{0}$. We obtain

$$
\boldsymbol{V}_{1}^{(1)}\left(r, t_{n}\right) \cong V_{10} \hat{z}-V_{10} \exp \left\{-\eta\left[1-\left(1+\xi^{2}\right)^{-2.65}\right]\right\}\left[\alpha_{n}\left(1+\xi^{2}\right)^{-1}(\hat{z}-\xi \hat{\boldsymbol{r}})+\beta_{n} \hat{z}\right]
$$

where $\alpha_{n}=\left[v_{c} /\left(v_{i n}+v_{c}\right)\right]\left(e E_{0} t_{n} / V_{10} m_{n}\right)\left(n_{e 0} / n_{n}\right)$ and $\beta_{n}=\left(n_{e 0} / n_{n}\right)\left(v_{c} t_{n}\right)\left[1+v_{i n} /\left(v_{i n}+v_{c}\right)\right]$. Thus the deflected flow has spatially dependent deflection angle $\theta^{\prime}$ and Mach number $M_{1}$, which are obtained from (9) to be $\theta^{\prime}(r)=\tan ^{-1}\left[V_{1 r}(r) / V_{1 z}(r)\right]$ and $M_{1}(r)=\left\{\left[V_{1 r}(r)^{2}+V_{1 z}(r)^{2}\right]^{1 / 2} / V_{10}\right\} M_{10}$, where $M_{10}$ is the Mach number of the unperturbed flow.

\section{Numerical Example}

Consider a case comparable with the experiment to be presented in the next section, the parameters of the incoming flow used in the numerical calculations are: $M_{10}=2.5, n_{n} \sim 10^{25} \mathrm{~m}^{-3}$ (i.e., $P_{1}=0.175 \mathrm{~atm}$ and $T_{1}=135 \mathrm{~K}$ ), $V_{10}=570 \mathrm{~m} / \mathrm{s}$, and $v_{c} \cong 2 v_{\text {in }} \cong 2.16 \times 10^{9} \mathrm{sec}^{-1}$.

The deflection angle $\theta^{\prime}(r)$ and the Mach number $M_{1}(r)$ of the deflected flow vary with the intensity of the discharge (depending on the applied electric field intensity and gauged by the maximum electron density $\left.n_{e 0}\right)$. Set $E_{0} \sim 10^{6} \mathrm{~V} / \mathrm{m}$ and $t_{n}=0.88 \times 10^{-5} \mathrm{~s}$ (i.e., $z_{0}=5 \mathrm{~mm}$ ), the parameters $\alpha_{n}$ and $\beta_{n}$ in (9) become $\alpha_{n}=0.334 \times 10^{-20} n_{e 0}$ and $\beta_{n}=0.253 \times 10^{-20} n_{e 0}$. Let $n_{e 0}=3 \times 10^{20} \zeta \mathrm{m}^{-3}$, where $\zeta$ is a variable parameter to weigh the discharge situation, so that $\alpha_{n}=\zeta$ and $\beta_{n}=$ $0.76 \zeta$. The two functions $\theta^{\prime}(r)$ and $M_{1}(r)$ are plotted in Figure 5(a) and Figure $5(\mathrm{~b})$ for the parameter $\zeta=0.23,0.2$, and 0.1 .

Using these results for each $\zeta\left(\right.$ i.e., $n_{0}$ ) as the parameters at the shock front location, the corresponding oblique angle $\beta_{c}(r)=\beta_{c}^{\prime}+\theta^{\prime}$ of the shock front can 


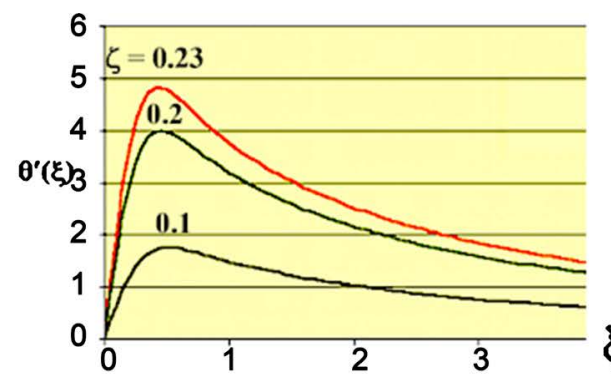

(a)

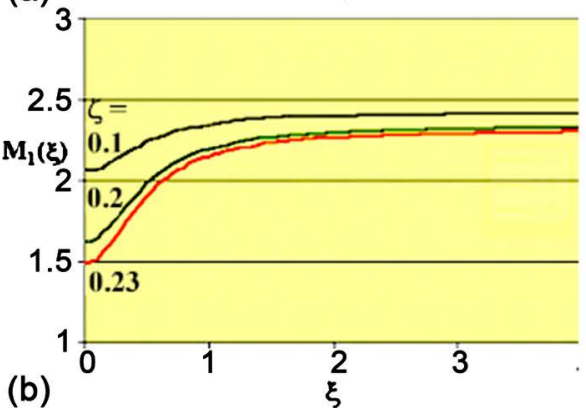

1.0

0.8

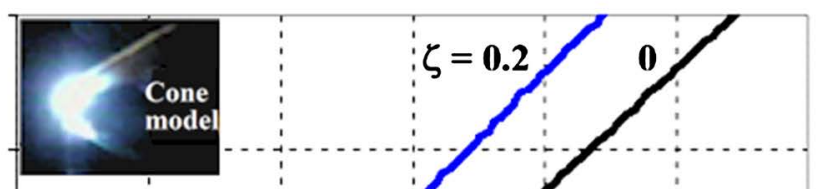

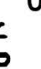

6

Shock front in
the presence

0.4 of plasma

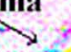

0.2

Baseline

shock front

0.0

0.0

(c)

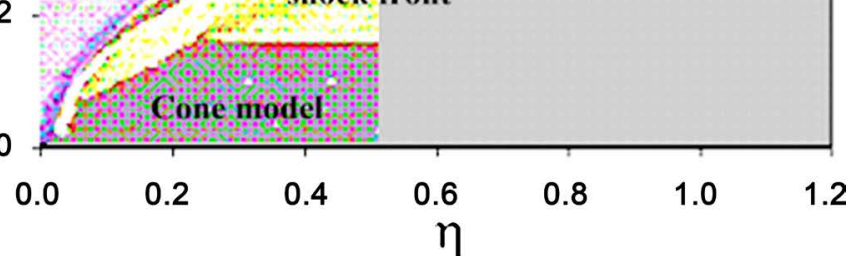

Figure 5. (a) $\theta(\xi)$, the deflection angle, and (b) $M_{1}(\xi)$, the Mach number, of an incoming flow after being scattered by a plasma spike; (c) attached shocks in a supersonic flow over a $60^{\circ}$ cone (represented by the shadow region) for two cases, $\zeta=0$ (no discharge) and $\zeta=0.2$ (corresponding to an intense discharge), where $\xi=r / z_{0}$ and $\eta=z / z_{0}$. The line labeled $\zeta=0$ represents the baseline shock front. The insert at left lower corner is a superimposed shadowgraph showing a baseline shock front and a shock front with angle increased by the plasma deflector shown in the insert at left upper corner, for comparison with the numerical results.

be determined by solving (3) iteratively to meet the condition that the normal component of the flow velocity on the cone surface $G^{\prime}\left(\theta_{c} ; \beta_{c}\right)=0$. Thus the position of the shock front can be determined by the trajectory equation

$$
\mathrm{d} z / \mathrm{d} r=\cot \beta_{c}=\cot \left(\beta_{c}^{\prime}+\theta^{\prime}\right)
$$

The result in the case of $\zeta=0.2$ (corresponding to a relatively intense discharge) is presented in Figure 5(c), in which the baseline shock front, having a shock angle $\beta_{0}=42.6^{\circ}$ (for $\theta_{c}=30^{\circ}$ ), is also presented for comparison. As shown, the shock angle is increased to $46^{\circ}$ by the plasma spike; agreeing well with the experimental result which is inserted at the left lower corner of the same figure for comparison. In this case, the peak electron density of the plasma deflector used in the numerical calculation is $n_{\mathrm{e} 0}=6 \times 10^{19} \mathrm{~m}^{-3}$, which agrees with that produced by the on-board diffused arc discharge, shown in the insert at the left upper corner of the same figure.

For each $\zeta$, a $\beta_{c}(\xi)$ distribution is determined. In terms of the determined $\beta_{c}(\xi)$ and $\delta$ for $\beta^{\prime}$ and $\delta^{\prime}$ in (2), one can obtain an equivalent Mach number distribution $M_{1 e q}(\xi)$. This is the Mach number distribution for an undeflected flow (i.e., in the absence of the plasma deflector) to generate the same shock structure as that in a plasma-deflected flow over the same cone. It is found that the effective Mach number $M_{1 e q}(\xi)$ of the incoming flow in the tip region has a similar spatial distribution as the corresponding $M_{1}(\xi)$ presented in Figure 5(b), and is smaller than $M_{10}$. 


\section{Experiment}

Experiment was conducted in a Mach 2.5 wind tunnel [17] [20] to study the effect of a plasma deflector on the shock wave structure discussed in Sec. 2. The upstream airflow had a flow speed $v=570 \mathrm{~m} / \mathrm{s}$, temperature $T_{1}=135 \mathrm{~K}$, and a pressure $P_{1}=0.175 \mathrm{~atm}$.

\subsection{Wind Tunnel Model and Electric Discharge}

Implement a plasma torch module [26] in a truncated-cone-shape wind tunnel model for on-board periodic electric discharges. This truncated-cone body is connected to a cylindrical body attached to a holder. The torch module consists of a sharpened solid tungsten rod of a diameter $d=2.4 \mathrm{~mm}$, as the central electrode, and a ceramic insulator which holds the tungsten rod in place concentrically with the truncated-cone body, as the outer electrode, to form the electrode pair for the discharge. The sharpened solid tungsten rod together with the ceramic insulator also mean to fill in the truncation of the cone, which recovers the model to a cone. A schematic of the model is presented in Figure 6(a). The truncated $60^{\circ}$ cone has a frontal diameter $D=11.1 \mathrm{~mm}$ and a height $L=12.7$ $\mathrm{mm}$. The cylindrical base of the cone has a diameter $D_{b}=25.4 \mathrm{~mm}$.

The gap between the tungsten rod and the inner wall at the front of the truncated-cone body is $3.5 \mathrm{~mm}$. The breakdown voltage is provided by a power supply sustaining $60 \mathrm{~Hz}$ periodic electric discharge. The nose of the model, shown in Figure 6(a), is a cone-shaped ceramic insulator with a short protruding spike, which replaces the truncated part of the cone. The distance from the tip to the edge of the truncated-cone surface is about $5 \mathrm{~mm}$. The $60 \mathrm{~Hz}$ power supply for periodic discharge operation is shown in Figure 6(b). During one of the two half cycles when the diode is forward biased, the capacitor is charged so the voltage across the electrodes is low and there is no discharge. During the other half cycle, the diode has a reverse bias. The charged capacitor increases the voltage across the electrodes and breakdown occurs. The discharge normally initiates in the region near the tip electrode where the applied electric field concentrates due to the cylindrical geometry. Therefore, it prefers the tip electrode to be negative so that the electrons can be pushed to the upstream region. Indeed,

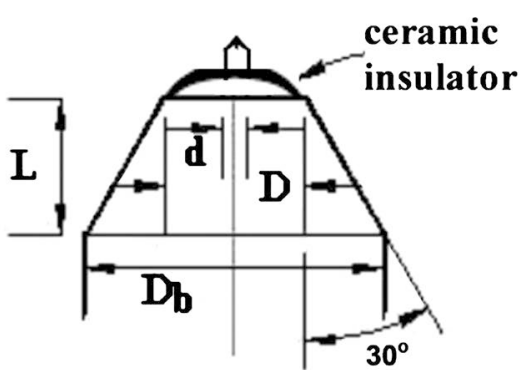

(a)

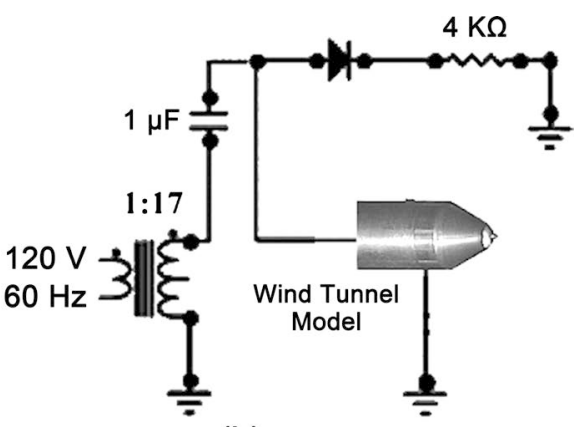

(b)

Figure 6. Schematics of (a) the model integrated with electrodes and (b) the circuit for 60 $\mathrm{Hz}$ periodic electric discharge. 
plasma effect on the shock wave structure has been observed only when the tip of the model is designated as the cathode.

The output voltage of the power supply has a peak of about $4.5 \mathrm{kV}$, exceeding the $4 \mathrm{kV}$ (for 5-mm gap) required for avalanche breakdown. The electric field intensity near the tip exceeds $1 \mathrm{MV} / \mathrm{m}$ before breakdown occurs. It reduces to a level less than $100 \mathrm{kV} / \mathrm{m}$ as the discharge current reaches the peak. The peak and average power of the discharge are about $1.2 \mathrm{~kW}$ and $100 \mathrm{~W}$, respectively. The maximum electron density of the discharge exceeds $10^{19} \mathrm{~m}^{-3}$.

A video camera, as the corresponding one to the CCD camera used to record the shadowgraph images of the flowfield, is used to record the spatial distribution and temporal evolution of the plasma glow with the frame rate of 30 frames per second and exposure time of $1 / 60 \mathrm{~s}$ (which is slightly less than six times of each discharge period). The video graph recorded in each frame is an integrated result over the exposure time, and thus the temporal variation of the plasma glow during a single discharge period cannot be recorded directly; but it can extract from the continuous video graph of the discharge. A sequence of 6 assembled video graphs showing the growth and decay of the plasma glow in the electric discharge, with a symmetrical spatial distribution with respect to the axis of a cone, are presented in Figure 7. In the video graphs, the flow is from right to left.

\subsection{Optical Diagnostics}

Shadowgraph method is used to optically diagnose the flowfield around the spike and nose of the cone. A black and white charge coupled device (CCD) camera, also with a frame rate of 30 frames per second and exposure time of 1/60 $\mathrm{s}$, is used to record directly the shadowgraph images of the flow structure. Although the starting times in recording each event (i.e., the starting time of each frame) by the two cameras are not synchronized, the events recorded by the two cameras can still be synchronized by counting number of frames from the reference frames, except, there will be a maximum possible time difference that is half of the exposure time (i.e., 1/120 s). The images extracted from videos recording the shadowgraph images of the flow and recording the plasma glow can provide the correlation between the strength of the plasma deflector and the degree of the shock structure modification; and any consistent relationships appearing in the correlation are useful for determining the plasma conditions, in order to achieve significant plasma effect on the shock wave.

\subsection{Experimental Results}

The spray-like plasma, generated by the electric discharge, acted as a spatially distributed plasma deflector, which was used to deflect the incoming flow. A video camera was used to record the shadowgraph images of the flow. The modification effect depended on the density, volume, spatial distribution, and location of the plasma deflector produced by the electric discharge, which varied in 


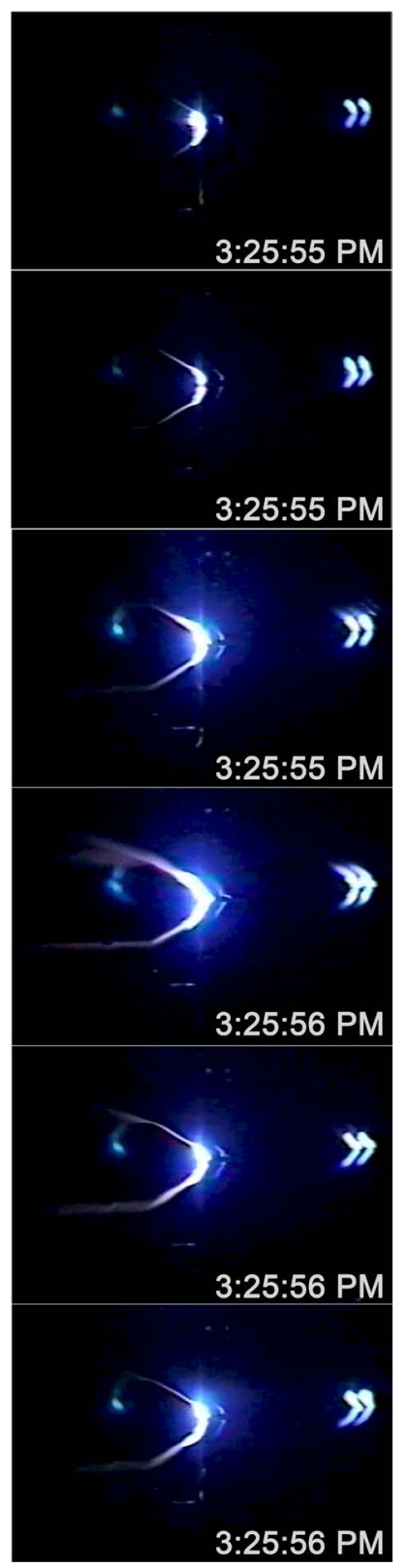

Figure 7. An assembled time sequence of six video graphs showing the growth and decay of the plasma glow during one discharge period in the middle of a wind tunnel run at Mach 2.5.

time; thus the plasma deflector increased its size and intensity from a low level to the maximum and then decayed to a low level, as demonstrated in Figure 7. This time varying behavior could cause the shock wave to also vary in time. However, the exposure time of each video frame is longer than each discharge period, the temporal variation of the shock wave structure during a single discharge period could not be recorded directly; the desired information regarding the temporal variation of the flowfield during the discharge was extracted from the continuous video graph of the flow. This is demonstrated in Figure 8, which includes a sequence of six shadowgraphs showing the responses of the incoming 


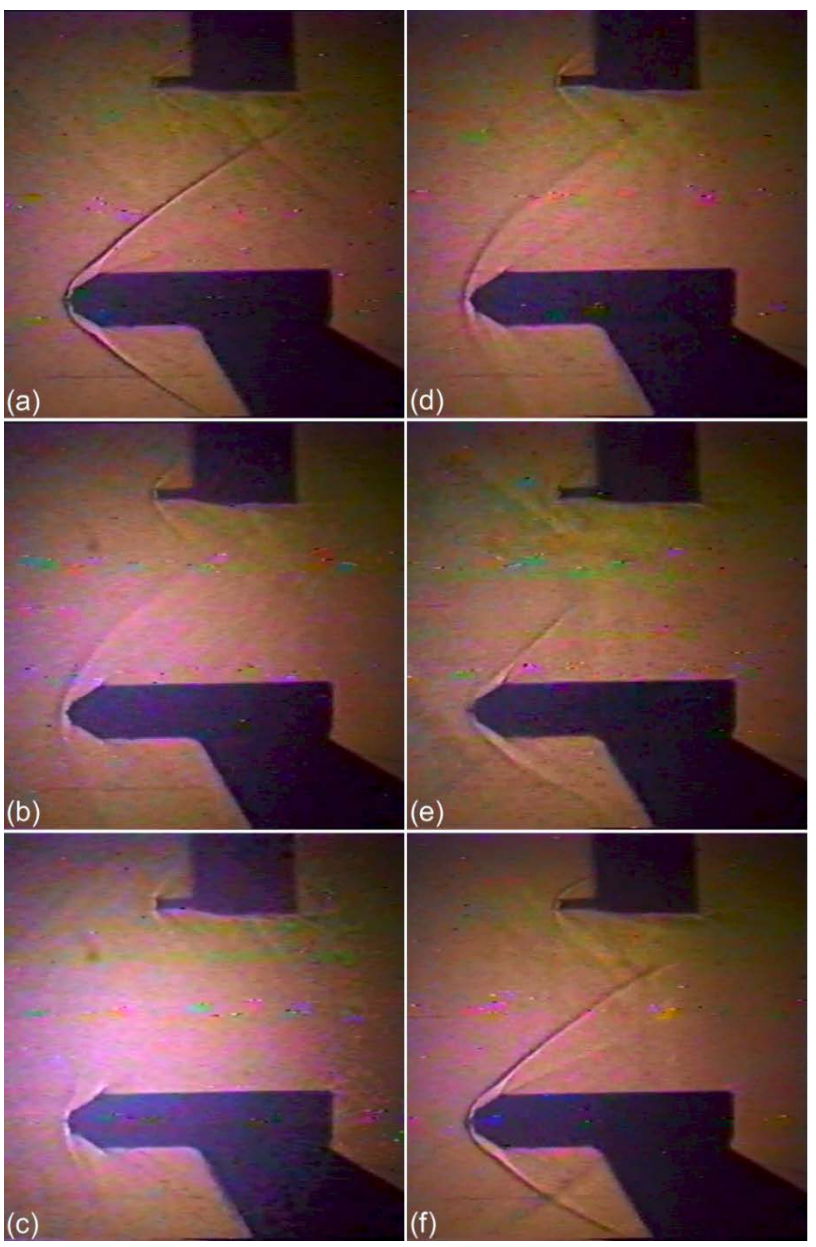

Figure 8. An assembled time sequence of six shadowgraphs (a)-(f) to represent the flow response to the plasma deflector during one discharge period in the middle of a wind tunnel run at Mach 2.5 .

flow to the growth and decay of the plasma deflector in one discharge cycle. In these shadowgraphs, the flow is from left to right.

The growth of the plasma deflector is manifested by the variation of the background brightness in the shadowgraphs. First, shadowgraph shown in Figure 8 (a) is in the case that the discharge just starts Therefore, the shadowgraph is darker (absence of background light from the plasma) and is similar to the baseline one. The next two presented in Figure 8(b) and Figure 8(c) correspond to the situation that the discharge is intensifying to reach the peak. As shown in Figure $8(\mathrm{~b})$, the intensified discharge first moves the baseline shock front upstream to become a detached curved shock front. As the plasma deflector is further intensified to reach the strongest level, its modification effect on the shock structure also reaches the maximum. The curved shock front in Figure 8(c) is much diffused, with larger curve angle and weaker appearance. As the intensity of plasma deflector decreases from the maximum level, the shock front also recovers toward to the baseline in the sequence of Figure 8(d) to Figure 8(f). A well-defined curve shock front reappears in Figure 8(d). It moves downstream 
to become an attached oblique shock as shown in Figure 8(e), but the shock front appears to be weaker than that of the baseline. The baseline shock front reappears in Figure 8(f) as the discharge is reduced to low level again. The recovery of the baseline shock front presented in Figure 8(f) indicates the steady of the incoming flow during the discharge cycle. These observations on unsteady shock motion containing the aforementioned features of the flowfield are typical of all the experiments performed and seen in the recorded videotape.

The pronounced influence of plasma on the shock structure is demonstrated by the result shown in Figure 8(c). A comparison of Figure 8(c) and Figure 8(a) clearly observes a transformation of the shock from a well-defined attached shock into a diffused and highly curved shock structure having a larger shock angle. This modification is an indication of shock wave being weakened by this plasma deflector. Using non-thermal plasma to mitigate shock wave [17] [20] is an ultimately desirable result that leads to the reduction of wave drag and shock noise at supersonic speeds; significantly improving supersonic flight.

\section{Summary}

A theory based on the deflection of the incoming flow by a symmetrically distributed plasma deflector in front of the shock as the process to modifying the shock wave structure has been formulated. Flow deflection increases the equivalent cone angle, which in turn increases the attached oblique shock wave angle. Moreover, when this equivalent cone angle exceeds a critical angle, the shock becomes a detached curved shock.

Wind tunnel experiments were conducted to explore the non-thermal plasma effects, based on plasma deflection theory, on the shock wave structures. A cone-shaped model was used as the shock generator and was facilitated with electrodes for on-board $60 \mathrm{~Hz}$ periodic discharge to generate plasma in front of the model. The tip of the central electrode in the model was shaped to match the cone angle and to enhance the electric field intensity in the region in front of the tip. The central electrode was set as the cathode. This arrangement together with the favorable electric field distribution made electron flow in the discharge much easier to pass through the shock front into the upstream (lower pressure) region before returning to the body of the model, set as the anode. As shown in Figure 7, the plasma glow of the discharge extends to the upstream region and has a symmetric distribution around the central electrode, which were found to be the necessary conditions to achieve noticeable plasma mitigation of shock waves.

As shown in the sequence of 6 shadowgraphs presented in Figure 8, the introduced plasma caused the shock front to have increased angle and dispersion in its structure as well as standoff distance from the model to become detached curved shock.

The theoretical deflection model is analyzed numerically. As demonstrated in Figure 5(c), the numerical results are in good agreement with the experimental 
results that the plasma deflector increases the oblique shock wave angle. The experimental results presented in Figure 8 also demonstrate that the plasma deflector can indeed convert an attached oblique shock to a detached curved shock, as indicated by the theory.

\section{Concluding Comments}

The wave drag and noise of the shock on the cone depends on the strength of the shock, which in turn depends on the Mach number of the flow as well as the shock wave structure. It is found that the effective Mach number $M_{1 e q}(\xi)$ of the deflected flow in the tip region is smaller than the unperturbed one $\left(M_{10}\right)$. A decrease in the effective Mach number of the incoming flow in the tip region verifies that the plasma deflector can indeed reduce the wave drag of the shock on a supersonic aircraft. Moreover, as the modified shock structure moves upstream away from a supersonic aircraft and becomes diffusive, it also results to the reduction of the wave drag on the supersonic aircraft and of the shock noise generated in the flow.

\section{Acknowledgements}

The author is grateful to Dr. D. Bivolaru for collaborative work. This work was supported in part by the Air Force Office of Scientific Research Grant AFOSRFA9550-04-1-0352.

\section{Conflicts of Interest}

The author declares no conflicts of interest regarding the publication of this paper.

\section{References}

[1] Anderson Jr., J.D. (1990) Modern Compressible Flow. McGraw-Hill, New York.

[2] Chang, P.K. (1970) Separation of Flow. Pergamon, Oxford.

[3] Batdorf, S.B. (1972) Alleviation of the Sonic Boom by Thermal Means. Journal of Aircraft, 9, 150-156. https://doi.org/10.2514/3.58947

[4] Levin, V.A. and Taranteva, L.V. (1993) Supersonic Flow over Cone with Heat Release in the Neighborhood of the Apex. Fluid Dynamics, 28, 244-247. https://doi.org/10.1007/BF01051214

[5] Riggins, D., Nelson, H.F. and Johnson, E. (1999) Blunt-Body Wave Drag Reduction Using Focused Energy Deposition. AIAA Journal, 37, 460-464. https://doi.org/10.2514/2.756

[6] Schülein, E. and Zheltovodov, A. (2011) Effects of Steady Flow Heating by Arc Discharge Upstream of Non-Slender Bodies. Shock Waves, 21, 383-396. https://doi.org/10.1007/s00193-011-0307-1

[7] Markhotok, A. (2015) A Mechanism of Wave Drag Reduction in the Thermal Energy Deposition Experiments. Physics of Plasmas, 22, 063512. https://doi.org/10.1063/1.4922434

[8] Gordeev, V.P., Krasilnikov, A.V., Lagutin, V.I. and Otmennikov, V.N. (1996) Plas- 
ma Technology for Reduction of Flying Vehicle Drag. Fluid Dynamics, 31, 313-317. https://doi.org/10.1007/BF02029693

[9] Baryshnikov, A.S., Basargin, I.V., Dubinina, E.V. and Fedotov, D.A. (1997) Rearrangement of the Shock Wave Structure in a Decaying Discharge Plasma. Technical Physics Letters, 23, 259-260. https://doi.org/10.1134/1.1261837

[10] Kuo, S.P., Kalkhoran, I.M., Bivolaru, D. and Orlick, L. (2000) Observation of Shock Wave Elimination by a Plasma in a Mach-2.5 Flow. Physics of Plasmas, 7, 13451348. https://doi.org/10.1063/1.873776

[11] Kuo, S.P. and Bivolaru, D. (2001) Plasma Effect on Shock Waves in a Supersonic Flow. Physics of Plasmas, 8, 3258-3264. https://doi.org/10.1063/1.1376422

[12] Appartaim, R., Mezonlin, E.D. and Johnson III, J.A. (2002) Turbulence in Plasma-Induced Hypersonic Drag Reduction. AIAA Journal, 40, 1979-1983. https://doi.org/10.2514/2.1559

[13] Bivolaru, D. and Kuo, S.P. (2002) Observation of Supersonic Shock Wave Mitigation by Plasma Aero-Spike. Physics of Plasmas, 9, 721-723. https://doi.org/10.1063/1.1433662

[14] Bivolaru, D. and Kuo, S.P. (2005) Aerodynamic Modification of Supersonic Flow around Truncated Cone using Pulsed Electrical Discharges. AIAA Journal, 43, 1482-1489. https://doi.org/10.2514/1.7361

[15] Kuo, S.P. and Bivolaru, D. (2007) The Similarity of Shock Waves Generated by a Cone-shaped Plasma and by a Solid Cone in a Supersonic Airflow. Physics of Plasmas, 14, 023503.

[16] Joussot, R. and Viviana Lago, V. (2016) Experimental Investigation of the Properties of a Glow Discharge Used as Plasma Actuator Applied to Rarefied Supersonic Flow Control Around a Flat Plate. IEEE Transactions on Dielectrics and Electrical Insulation, 23, 671-682. https://doi.org/10.1109/TDEI.2015.005327

[17] Kuo, S.P. (2007) Plasma Mitigation of Shock Wave: Experiments and Theory. Shock Waves, 17, 225-239. https://doi.org/10.1007/s00193-007-0112-Z

[18] Sun, Q., Cheng, B.Q., Li, Y.H., Kong, W.S., Li, J., Zhu, Y.F. and Jin, D. (2013) Computational and Experimental Analysis of Mach 2 Air Flow over a Blunt Body with Plasma Aerodynamic Actuation. Science China Technological Sciences, 56, 795-802. https://doi.org/10.1007/s11431-013-5177-6

[19] Exton, R.J., Balla, R.J., Shirinzadeh, B., Brauckmann, G.J., Herring, G.C., Kelliher, W.C., Fugitt, J., Lazard, C.J. and Khodataev, K.V. (2001) On-Board Projection of a Microwave Plasma Upstream of a Mach 6 Bow Shock. Physics of Plasmas, 8, 5013-5017. https://doi.org/10.1063/1.1407819

[20] Kuo, S.P. (2016) Air Plasma Mitigation of Shock Wave. Advances in Aerospace Science and Technology, 1, 59-69. https://doi.org/10.4236/aast.2016.12006

[21] Brown, S.C. (1967) Basic Data of Plasma Physics. $2^{\text {nd }}$ Edition, MIT Press, Cambridge.

[22] Kuo, S.P. and Kuo, S. (2005) A Physical Mechanism of Non-Thermal Plasma Effect on Shock Wave. Physics of Plasmas, 12, Article ID: 012315.

[23] Kuo, S.P. (2004) Conditions and a Physical Mechanism for Plasma Mitigation of Shock Wave in a Supersonic Flow. Physica Scripta, 70, 161-165. https://doi.org/10.1088/0031-8949/70/2-3/014

[24] Kuo, S.P. (2005) Shock Wave Modification by a Plasma Spike: Experiment and Theory. Physica Scripta, 71, 535-539. https://doi.org/10.1238/Physica.Regular.071a00535 
[25] Kuo, S.P. and Kuo, S. (2006) Theoretical Study of Plasma Effect on a Conical Shock Wave. Physics of Plasmas, 13, Article ID: 033505.

[26] Kuo, S.P., Koretzky, E. and Orlick, L. (2001) Methods and Apparatus for Generating a Plasma Torch. United States Patent, US 6329628 B1. 